\title{
A REGION-BASED APPROACH TO BUILDING DETECTION IN DENSELY BUILD-UP HIGH RESOLUTION SATELLITE IMAGE
}

\author{
Zongying Song, Chunhong Pan, Q Yang* \\ National Laboratory of Pattern Recognition, Institute of Automation \\ Chinese Academy of Sciences, Beijing, 100080, China \\ \{zysong, chpan, qyang\}@nlpr.ia.ac.cn
}

\begin{abstract}
We propose a novel region-based approach for building detection in high-resolution satellite image with densely build-up buildings. In our method, first the prior building model is constructed with texture and shape features from training building set. After oversegmentation of input image into many small regions, we identify regions which have a similar pattern with prior building model. These regions are called building like regions (BLRs). Then we group BLRs to get candidate building regions (CBRs), which have similar shape with prior building model. Next, lines which have strong relationship with each CBR are extracted. From these lines and CBR boundaries, 2-D rooftop hypotheses are generated. At last, shadows and geometrical rules are used to verify the hypotheses. Experimental results are shown on area with hundreds of buildings.
\end{abstract}

Keywords: Remote sensing, Image region analysis, Object detection

\section{INTRODUCTION}

Automatic extraction of man-made objects from high resolution satellite images is a fundamental task in image understanding. In particular, extraction of buildings have become the most important one because of its numerous applications in urban mapping, planning and GIS update. For such applications, researchers in the last two decades have proposed a number of approaches [1][2]. Kim and Muller used a graph-based detection technique to extract 2D building outlines and used stereo image pairs to extract height information [3]. Noronha et al. proposed a hypothesis and verification method for building extraction [4]. First, the 2D building roof hypotheses are generated from linear features by perceptual grouping, and those hypotheses are then verified by $3 \mathrm{D}$ evidence from shadows and walls of building.

But until now building detection still remains an open problem. Most current approaches do not work in densely build-up areas, and results for data sets involving several hundred or thousand buildings have not been shown [1]. There are a number of challenges in this problem. The first difficulty is that the image is a 2-D projection from the real 3D world and hence inherently ambiguous. Secondly, shadow and occlusions frequently occur, which makes appearance of buildings in image incomplete and irregular. Thirdly, there are many kinds of environment noises such as roads, trees and squares which are not easy to distinguish from buildings. At last, in high resolution satellite images the buildings have many detailed and complex structures. They may appear very differently in intensity levels, shapes

${ }^{*}$ The work was supported by 863 Program of China, Grant No: 2005AA114130. and other patterns. There are nearly no general features to describe buildings. Therefor, a general classification approach for all building class will inevitably fail. It should be reasonable and practical to propose a building detection method only for one specific building class.

To deal with the above difficulties, researchers have proposed many approaches [3][4] [5], which usually use lines and junctions. However, these methods are not suitable for building detection in densely build-up high resolution satellite image. Because in the high resolution satellite image with hundreds of complex buildings, the number of lines or junctions is huge, and also there are a large number of noise which is very hard to distinguish from the real information we needed. In this paper, we focus on regions. Because region-based methods have some significant advantages compared to methods based on lines or junctions. First, some prior high-level knowledge, such as texture or intensity models, shape models can be incorporated into the region-based method. Secondly, lines or junctions can be effectively and simply be used in the region-based method.

Segmentation is one of the key area in computer vision and pattern recognition society. There are many traditional schemes for image segmentation. Several approaches have been proposed to segment the objects by using prior information about the shape of specific, which are called shape based segmentation. Most of these methods decompose objects into parts by measuring the shape similarity between image data and an arrangement of predefined part models [6]. From the other view of point, the building detection problem can also be regarded as an image segmentation problem. The difference between shape based segmentation problem and the building detection problem is that building detection should not only use prior shape information but also some other information about building. We can extract buildings by measuring the similarity between the image data and the prior building model. The work we need to do is that we should construct a prior building model from many aspects, such as shape, texture, intensity.

Based on the above analysis, we propose a region-based building detection approach using the prior building model. The whole approach can be divided into four stages. In the first stage, we construct prior building model using texture and shape features from the training building set. To utilize shape information effectively, we designed new shape descriptors to describe the regularity of a shape. In the second stage, we over-segment the input image into many small regions. Given the prior model and the over-segmented image, the regions which have a similar energy distribution with the prior model are identified. We call these regions building like regions (BLRs). In the third stage, we group the BLRs together to generate region groups which have a similar shape with the prior build- 
ing model, we call these region groups candidate building regions (CBRs). For this special purpose, we proposed a seed based region grouping method which is simple and fast. In the last stage, we extract lines which have strong relationship with each CBR. From these lines and the CBR boundaries, we generate 2-D rooftop hypotheses. Finally, neighborhood relationship, shadows and some geometrical evidences are used to verify the hypotheses. Fig. 1 shows the flow of the whole strategy.

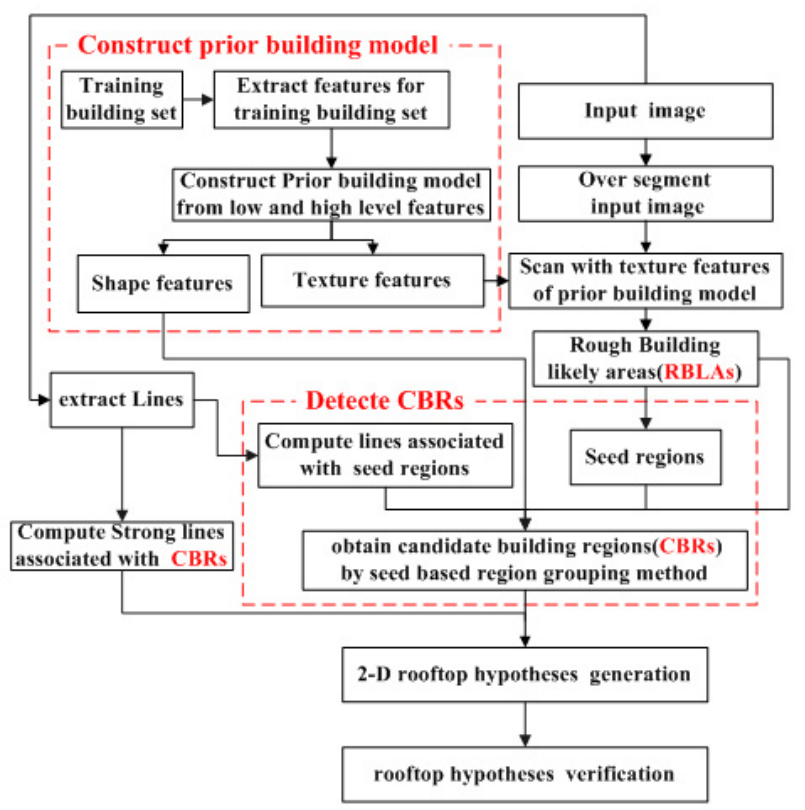

Fig. 1. The flow of our approach. The two frames (in dashed red lines) are respectively denote the prior building model construction stage and the CBRs detection stage.

In the following sections, we describe the stages gradually. Section 2 will illustrate stage 1 , section 3 will explain stage 2 and 3 , section 4 will show the last stage, and section 5 will describe the experiment results and a brief conclusion of the proposed method.

\section{FEATURES AND PRIOR BUILDING MODEL}

In this section we will construct a prior building model over the texture and shape features from training building set. The training building set in our experiment is 120 buildings in rectangular shape which are manually selected from the whole image of an urban area.

Intensity and texture are widely used low-level features to describe regions. But intensity is not suitable to describe building regions, since buildings may have very different materials and structures which will lead to fully different intensity levels. On the other hand, texture features are much more appropriate. Buildings are more regular than natural objects, hence, most energy of buildings will mainly distribute on low-level frequency-band and its high-level frequency information is limited. Fig. 2 shows the experimental result with the three-level wavelet decomposition for building regions and natural regions. From this figure, one can observe that the energy distribution histogram is a salient feature to distinguish buildings from natural objects.

Shape is used as the other important feature in our algorithm. Many methods have been proposed to describe shape, however, they

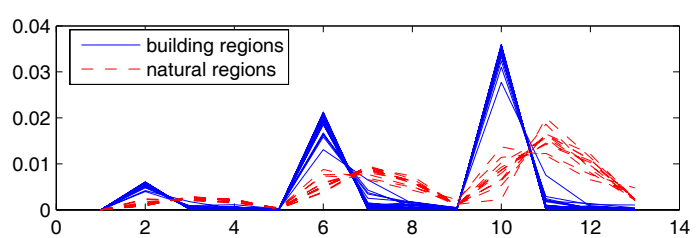

Fig. 2. Energy distribution histograms by three-level wavelet decomposition. The real blue line and the dashed red line respectively denote the energy distribution histograms of building regions and natural object regions.

are either too complex or too expensive to be computed. For the building detection problem, we proposed a new method to describe the regularity of a building shape. We define a series of discrete contour descriptors. A contour descriptor is a $3 \times 3$ window (pixel), and the pixel of its center resides on the contour of a region. Fig. 3 shows the 4 descriptors used in our method.On these descriptors many shape regularity measurements can be defined, for example, the distribution histogram on these descriptors.

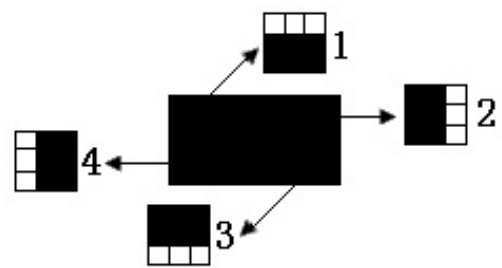

Fig. 3. Four contour descriptors used to measure the regularity of a shape.

Here, buildings with rectangular shape are selected as our building class. For this building class, two shape features are used: one is the rectangle ratio, the other is the contour descriptors ratio which is the total number of the 4 contour descriptors ( 3 ) divided by the number of contour pixels.

We extract the energy distribution histogram for the 120 training buildings, and then compute the mean energy distribution histogram as the texture feature of the prior building model. For the shape feature of the model, we simply use the above two shape features for rectangular shape.

\section{CANDIDATE BUILDING REGIONS(CBRS) RECOGNITION}

In this section CBRs will be obtained from the input image in two stages. Before computation of the CBRs, there are two preprocessing tasks. The first is to over-segmented the input image $I$ into many small regions by mean-shift method [7]. We denote $I=\bigcup_{i=1}^{n} v_{i}$, where each $v_{i}$ is a small region. The second preprocessing work is to extract lines from the input image $I$ by method in [8].

\subsection{Identification of BLRs}

We scan the whole over-segmented image to compute the similarity of the energy distribution histogram between each small region and the prior building model. The similarity of two distributions are computed by KL-divergence. After that we will get a similarity 
measurement $\theta_{i}$ for each small region $v_{i}$. Next, we use a threshold method to decide which region is a building like region (BLR). Fig. 4 is the BLR image of the input image (Fig. 7). From Fig. 4 one can find that almost all regions of buildings are included in this image and most non-building regions have been eliminated. Although a little noise have been include in the BLR image, about half of the oversegmented regions have been eliminated. This has greatly decreased the computation complexity and simplified the whole process.

The KL-Divergence is the probability distribution similarity measurement function:

$$
K L(p \| q)=\sum_{x \in \chi} p(x) \log \frac{p(x)}{q(x)}
$$

where $K L(p \| q)$ is the kl-divergence equation of discrete form, $p$ and $q$ are two probability distributions. Because the KL-Divergence is not symmetrical, the final equation we used for energy distribution similarity measurement is:

$$
M(p \| q)=\frac{1}{2}(K L(p \| q)+K L(q \| p))
$$

where $p$ and $q$ are normalized energy distribution histograms.

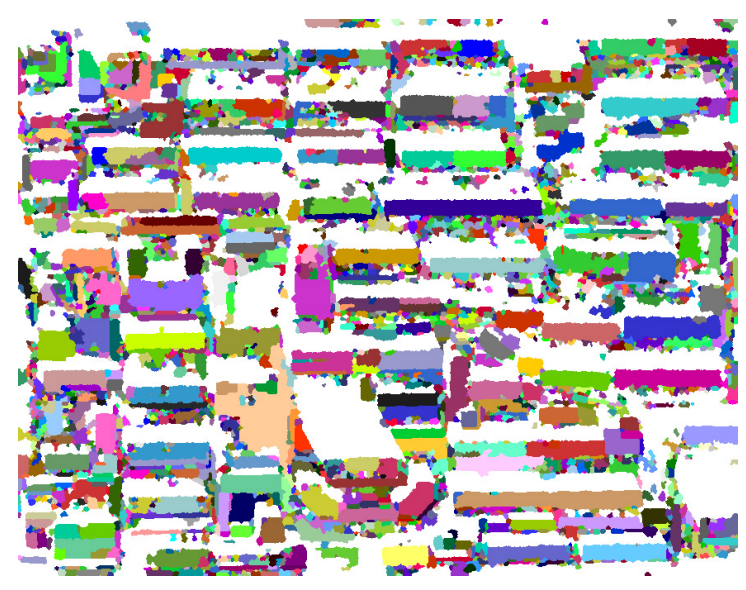

Fig. 4. Building like regions (BLRs) image. The color used in this image are randomly generated and allocated for each region, which are only used to display the result conveniently.

\subsection{Candidate building regions detection}

We can find that in Fig. 4, a building area has been over segmented into many small regions. Therefor, in this section we will group these small regions to generate a full building region. In our method, region grouping process is guided by the shape of prior building model. In other words, the objective of region grouping is to generate region groups which have similar shape with prior building model. These region groups are called candidate building regions (CBRs).

There are many region grouping methods, but most of them are complex and computationally expensive, making them unsuitable for building detection. From the observation of real buildings, we can find that although buildings often have various shape and many detailed structures, most part of a building is smooth which is easily recognizable. So we regard these smooth regions as the seed of a building. These seeds are used as the heuristic information for region grouping. From the above analysis, we propose a region grouping method starting from these seed regions. The idea is that the region group for a building should be in the small neighborhood of its seed region. Therefore, the CBR grouping problem can be transferred to find region groups which have a more similar shape with prior building model in a small neighborhood of seed regions. Because the number of small regions in a building should be very small, we can easily compute every possible connected region group in the local neighborhood of a seed region. After obtaining all the region groups, it will be very easy to compute the region group which is most similar with the prior building shape.

However, the two shape features for rectangular shape building class is not rotation invariant, so the direction of each candidate building should be computed. Here we just take the direction of the seed region to be the direction of a CBR group. We propose a method to compute the direction of the seed region by lines.

First, lines near or in the seed regions are extracted, and then these lines are divided into different groups by their directions. The mean direction of the lines in each group are computed as a candidate direction for the seed region. After the above computation, several candidate directions are obtained for each seed region. For each candidate direction $\alpha$, we rotate the original coordinate system counterclockwise to align it with $\alpha$. In the new coordinate system, the shape discriminator $S\left(v_{i}\right)=c_{1} \cdot R_{r}\left(v_{i}\right)+c_{2} \cdot R_{d}\left(v_{i}\right)$ is computed, where $S\left(v_{i}\right)$ is the shape discriminator of the region $v_{i}, c_{1}$ and $c_{2}$ are the weight of the respective shape features, $R_{r}\left(v_{i}\right)$ is the rectangle ratio of the region $v_{i}$, and $R_{d}\left(v_{i}\right)$ is the contour descriptors ratio of the region $v_{i}$. The direction which maximizes the shape discriminator $S\left(v_{i}\right)$ will be chosen as the direction of the seed region.

\section{Seed based region grouping method}

Input: a seed region $v$ and its direction $\alpha$, three-level (Fig. 5) neighbors set $N$ of $v$.

Output: a region group.

1. Counterclockwise rotate the origin coordinate system to align it with $\alpha$.

2. Construct a nearest-neighbor graph $G$ of the seed region $v$ and its neighbor set $N$.

3. Find all the connected subgraph $G_{i}$ containing $v$ in $G$, let $S R_{i}$ be the vertex set of each subgraph $G_{i}$.

4. Compute the shape discriminator in the new coordinate system for each $S R_{i}$, the one corresponding to the maximal shape discriminator will be the result region group.

5. Merge the small regions in the result region group to generate a CBR for seed region $v$.

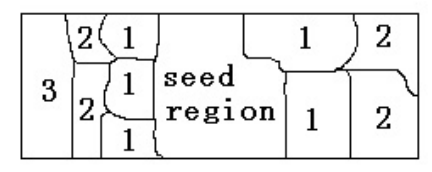

Fig. 5. Different level of neighborhood, regions labeled by 1 are the first-level, regions labeled by 1 and 2 are second-level, regions labeled by 1,2 and 3 are third-level.

The Fig. 6 has shown the CBR detection result by the seed based region grouping method. Many small irregular regions have been grouped into regular shapes. Especially, the building regions with 
different intensity levels and detailed structures have been correctly grouped. Also we can see that our method is rotation invariant.

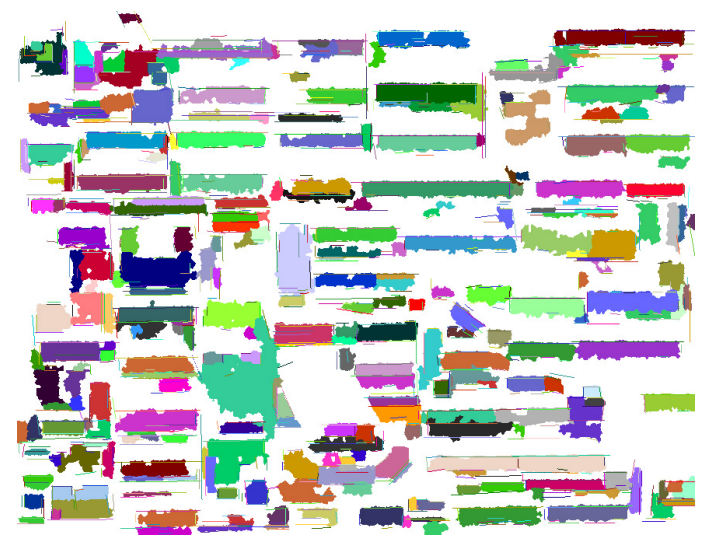

Fig. 6. Candidate building regions with strong lines. The color used in this image are randomly generated and allocated for each region and line, which are only used to display the result conveniently.

\section{2-D ROOFTOP HYPOTHESES GENERATION AND VERIFICATION}

2-D rooftop hypotheses of buildings are generated by the shape of prior building model. In this paper, we just need to generate an rectangular shape for each candidate building regions(CBRs).

After getting the CBRs, we extract the strong lines for each CBR(Fig. 6). The strong line is parallel or perpendicular with the CBR and near the boundary of the CBR. Next, we integrate the boundary of CBRs and these strong lines to generate a 2-D rectangular rooftop hypothesis for each CBR.

In the hypotheses verification stage, the topological relationship between buildings and their shadows are used as the main evidence to identify these hypotheses. Another evidence includes the typical geometrical size of building.

\section{RESULT AND CONCLUSION}

Fig. 7 shows experimental result of our approach. The test image is densely build-up and the buildings in this image have many detailed structures and appeared very different. From the ground truth given manually, there are nearly 100 rectangular buildings in this test image. It has to be noted that human cannot perfectly recognize all the buildings in rectangular shape because of various shadows, blurring and occlusion complications. In our result, 100 buildings(in green) are all correctly detected, only one noise region(in yellow) is detected as building. Among the detected buildings, about 7 buildings (in red) are partially reconstructed, and about 5 buildings (in blue) are merged with noise regions. Although the buildings have very complex patterns and structures, $100 \%$ buildings have been correctly detected, and nearly $90 \%$ buildings have been correctly reconstructed. The excellent results demonstrate the effectiveness and validity of our algorithm.

It is very challenging to extract buildings from such a complex scene with such a large number of buildings. The performance of our method is promising. We think the good performance of our approach comes from: first, our method is designed for only one shape building class. Secondly, construction of prior building model make

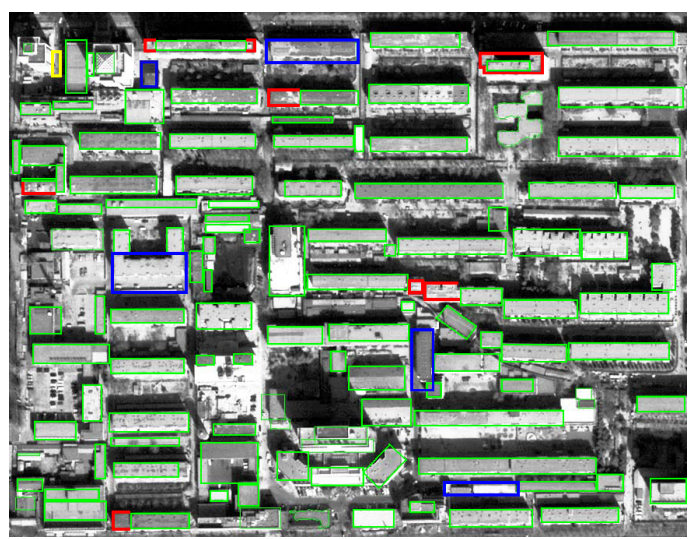

Fig. 7. Experimental result of test image. Green frame: building detection and reconstruction results; Red frame: detected buildings which are not completely reconstructed; Blue frame: detected buildings which are merged with noise; Yellow frame: noise regions which are detected as buildings.

our method take use of prior knowledge. Thirdly, the texture and shape features we have selected and designed are very appropriate for building detection problem.

However, there are still some limitations in our method. First, the detected results are dependent on the initial over-segmentation and line detection. Second, lines should be used in combination, and also all lines associated with CBRs should be considered. Finally, the building model is assumed to be simple, so buildings with complex shape and structures cannot be correctly reconstructed in our current algorithm. These will be our future research focus.

\section{REFERENCES}

[1] Helmut Mayer, "Automation object extraction from aerial imageryca survey focusing on buildings," CVIU, vol. 74, pp. 138149, May 1999.

[2] J.B.Mena, "State of the art on automatic road extraction for gis update," Pattern Recognition Letters, vol. 24, pp. 3037-3058, Dec. 2003.

[3] T.Kim and J-P. Muller, "Development of a graph-based approach for building detection," Image and Vision Computing, vol. 17, pp. 3-14, Jan. 1999.

[4] S.Noronha and R. Nevatia, "Detection and modeling of buildings from multiple aerial images," IEEE Trans. Pattern Anal. Machine Intell., vol. 23, pp. 501-518, May 2001.

[5] ZuWhan Kim and Ramakant Nevatia, "Automatic description of complex buildings from multiple images," CVIU, vol. 96, pp. 60-95, Oct. 2004.

[6] A.EL-Baz, R.M.Mohamed, and A.A.Farag, "Shape-constraint for accurate segmentation in remote sensing imagery," Prof. of International Conference on Information Fusion, vol. 2, pp. 1154-1161, 2005.

[7] D.Comaniciu and P.Meer, "A robust approach toward feature space analysis," IEEE Trans. Pattern Anal. Machine Intell., vol. 24, pp. 603-619, May 2002.

[8] A.R. Hanson J.B. Burns and E.M. Riseman, "Extracting straight lines," IEEE Trans. Pattern Anal. Machine Intell., vol. 8, pp. 425-455, 1986. 